\title{
Review
}

\section{Guardians of the Cell: Effector-Triggered Immunity Steers Mammalian Immune Defense}

Thomas A. Kufer, ${ }^{1,4, \star}$ Emma M. Creagh, ${ }^{2,4, \star}$ and Clare E. Bryant ${ }^{3,4, \star}$

The mammalian innate immune system deals with invading pathogens and stress by activating pattern-recognition receptors (PRRs) in the host. Initially proposed to be triggered by the discrimination of defined molecular signatures from pathogens rather than from self, it is now clear that PRRs can also be activated by endogenous ligands, bacterial metabolites and, following pathogeninduced alterations of cellular processes, changes in the F-actin cytoskeleton. These processes are collectively referred to as effector-triggered immunity (ETI). Here, we summarize the molecular and conceptual advances in our understanding of cell autonomous innate immune responses against bacterial pathogens, and discuss how classical activation of PRRs and ETI interplay to drive inflammatory responses.

Back to the Future: the Concept of Innate Immunity and PRRs

In order to survive, all species have developed a means to defend themselves against invasion and colonization by pathogens. This generates a developmental arms race between hosts and pathogens, forcing the host to evolve detection mechanisms that are robust and specific. In mammals, two fundamental immune responses exist: the innate immune system, where cells can react towards pathogens by detecting conserved structures using germline encoded receptors, and the adaptive immune system, consisting of specialized cells that can rearrange and mutate their receptors to adapt to recognizing foreign substances. The seminal paradigm, coined by Charles Janeway, proposed that a family of receptors triggered by molecules on pathogens, termed pathogen-associated-molecular patterns (PAMPs) (see Glossary), activate innate immunity to discriminate self from non-self. This is now well proven experimentally and many PAMP receptors (PRRs), their ligands, signaling pathways, and effector mechanisms have been identified. In this work, we refer to the PRR-ligand-induced innate immune reaction, as classical induced innate immunity. With increased knowledge, so comes increased complexity. Many of the proposed PAMPs are also present in commensal bacteria, which do not induce strong immunity. This has led to the development of the concept of microbeassociated molecular patterns (MAMPs), which can be present in commensals as well as pathogens [1]. In order to avoid confusion of this semantic differentiation, we refer to any PRR-activating ligand as a PAMP.

Pioneering theoretical work by Matzinger proposed that the body could react to insults or danger signals (damage-associated molecular patterns; DAMPs) to mount inflammatory and adaptive immune responses [2]. DAMPs, including ATP, heat shock proteins, and nuclear DNA-binding proteins (among others), are all compartmentalized in the cell, only to be released upon pathogen-induced cell death or tissue destruction, and subsequently detected by DAMP receptors [3]. This showed that mammalian organisms are capable of pathogen detection directly by their PAMPs, but they also sense tissue damage induced by invasion and pathogen growth. Natural killer (NK) cells tolerate non-MHC-bearing hosts, and based on the behavior of these cells, Vivier and Pradeu recently proposed the discontinuity theory of immunity [4]. According

\section{Highlights}

Pathogen-mediated alterations in the cellular environment can contribute to mammalian innate immunity in a process referred to as effector triggered immunity (ETI).

Host-derived second messengers and metabolites are emerging as triggers of innate immune pathways.

Subcellular receptor localization, the cytoskeleton, and other cellular components can play key roles in mammalian ETI.

Coactivation of PRRs and ETI may be required for maximal activation of innate immunity.

${ }^{1}$ Institute of Nutritional Medicine, Department of Immunology, University of Hohenheim, Stuttgart, Germany ${ }^{2}$ School of Biochemistry and Immunology, Trinity Biomedical Sciences Institute, Trinity College Dublin, Dublin, Ireland ${ }^{3}$ Department of Veterinary Medicine, University of Cambridge, Cambridge, UK ${ }^{4}$ These authors contributed equally.

${ }^{*}$ Correspondence:

thomas.kufer@uni-hohenheim.de (T.A. Kufer), ecreagh@tcd.ie (E.M. Creagh), and ceb27@cam.ac.uk (C.E. Bryant). 
to their more philosophical view, the immune system might be activated by changes in the environment (or the cell), rather than by detection of defined molecules only [4]. Such discontinuity might represent the presence or absence of a particular PAMP or DAMP, but could also cover pathogen- or damage-initiated alterations in intracellular signaling pathways, thus leading to the activation of immunity.

In addition to DAMP sensing, indirect pathogen sensing was first identified in plants, where PAMP recognition by defined receptors - referred to as PAMP-triggered immunity (PTI) - was clearly distinguished from the recognition of pathogen effectors within the cell, or effector-triggered immunity (ETI) [102]. ETI is mediated, at least in some cases, by recognition of changes in cellular signaling pathways by host proteins that guard the cells. This scenario is referred to as the guard hypothesis [118]. In this review, we discuss recent advances suggesting that indirect recognition of pathogens is also implicated in mammalian immunity and overview similarities between plant and animal resistance mechanisms. The article presents a strong focus on cell-autonomous responses against bacterial pathogens, and highlights open questions in the field (see Outstanding Questions). Key discussion points include currently emerging concepts of innate immune detection, factors governing the activation of immune responses, and the importance of cytoskeletal rearrangements in the regulation of many of these responses.

\section{The Good, the Bad and the Ugly: Discrimination of Pathogens}

In view of the complex microbial communities that cohabit within the human body, how does the host sense the presence of microbes at all, and how does it discriminate between commensal bacteria and pathogens? Localization and activity of pathogens are likely to contribute to answering these important questions. In their patterns-of-pathogenesis hypothesis, Vance et al. suggested that cytosolic access, pathogen growth, and disruption of the host cytoskeleton are key triggers to discriminate pathogenic from nonpathogenic bacteria [5]. Does experimental evidence support such a hypothesis?

\section{Cytosolic Access}

Since the discovery of nucleotide-binding oligomerization domain-containing proteins (NOD) 1 and NOD2 as the first mammalian cytosolic immune sensors for bacterial invasion [6,7], it is clear that cytosolic sensors of invasive pathogens exist in most, if not all, cells of the body. Given the relatively recent identification of caspase-11 in mice, and caspase-4 and -5 in humans [binding intracellular lipopolysaccharides (LPS) to induce their activation] [8], it is likely that additional, unidentified sensors still exist. Many of the cytosolic sensors can also respond to pathogens that do not directly access the cytosol by sensing translocated PAMPs in the cytosol [9]. Although not the focus of this review, sensor mechanisms also exist for the detection of viruses [10].

\section{Pathogen Growth and Sensing}

The most efficient activation of $T$ cell responses usually occurs in response to live-attenuated vaccines, suggesting that specific patterns of live microbes can be sensed by the host -sometimes referred to as vita-PAMPs [11,12]. For example, bacterial mRNA was identified as being at least one of the components capable of enhancing innate immune responses and triggering adaptive immunity via Toll-like receptor (TLR)8 signaling in mice and pigs [13-15]. Another viable bacterial factor detected in the cytosol and produced by Gram-positive bacteria, is cyclic-di-adenosine monophosphate (cGAMP), which contributes to sensing of live bacteria by activation of the innate sensor stimulator of interferon genes (STING) in mouse bone-marrow-derived macrophages (BMDMs) [16]. Apart from this, bacterial cell division can lead to recruitment of host septins (a class of cytoskeletal filament proteins) that trigger xenophagy, and clearance of tagged bacteria [17].

\section{Glossary}

Absent in melanoma (AIM)2: a cytosolic DNA sensor.

Associated speck-like protein containing a CARD (ASC): adaptor protein in the inflammasome. Autophagy: process of active engulfment of material and pathogens in cytosolic double-membrane organelles, targeting these for degradation in phagolysosomes.

CpG DNA repeats: sequences in DNA that show a high density of $\mathrm{CPG}$ dinucleotides that can be targeted by DNA methylation.

Cyclic GMP-AMP synthase (cGAS): cytosolic sensor for cyclic dinucleotides. Damage-associated molecular pattern (DAMP): host molecules that can activate PRRs.

Discontinuity theory of immunity: theoretical model of how the immune system may be activated by changes in the cellular environment (rate of change in a stimulus), rather than by the stimulus itself.

Effector-triggered immunity (ETI):

immune cascade in plants triggered by a pathogen effector protein.

Familial Mediterranean fever (FMF): autoinflammatory disease associated with mutations in MEFV encoding pyrin.

Flagellin: bacterial protein recognized by TLR5 and the NLRC4 inflammasome; a main component of bacterial flagella.

Guard hypothesis: proposed guard mechanism of how plants can sense perturbation of signaling pathways by pathogen effector proteins. Interactions of effector pathogen proteins with host proteins, causes a change in their structure, subsequently recognized by the plant's guard R proteins. Innate immune memory (or trained immunity): cellular process involving epigenetic modifications, and leading to altered inflammatory responses following a previously encountered exposure to pathogen (memory). Legionnaires' disease: bacterial pneumonia caused by Legionella spp. Microbe-associated molecular pattern (MAMP): similar to PAMP; but is PRR-activating structure found in pathogenic and nonpathogenic microbes. NLRP1b: NACHT, LRR, FIIND, CARD domain and PYD domains-containing protein 1, NLR protein that forms an inflammasome. 
Disruption of the Host Cytoskeleton

Most bacterial pathogens either deliver effector molecules into the host cell and/or invade the cell directly. Various effector molecules (e.g., type III effector proteins from enteroinvasive bacteria) can change cellular processes to alter inflammatory signaling, induce pathogen uptake by the cell, trigger cell death, and/or enhance survival of infected host cells. Some common strategies used by bacterial pathogens include the disruption of membrane integrity by insertion of pore forming toxins (e.g., alpha-toxin and aerolysin from Staphylococcus aureus and hemolysin from Escherichia coli), or via cell invasion and perturbation of the F-actin cytoskeleton (see below) [18].

Aside from these cell-based aspects of detection, differentiation between a pathogen and commensal is also provided by the anatomical localization. In the intestine, for example, this is provided by physical barriers, including tight-linked epithelial cells, the mucus, and the action of a range of antimicrobial substances including siderophores, which capture growth promoting ions, antimicrobial peptides and immunoglobulins [19]. This barrier, however, is not a solid wall because it is highly adaptive in its repellence for microbes. Secretion of antimicrobial peptides such as the antibacterial lectin regenerating family member 3y (Regllly) in mice, for example, is controlled by classical induced innate immunity, and these peptides are enriched in the inner mucus layer to avoid colonization by microbes [20]. The capacity to overcome these barriers is a hallmark of pathogens. In conclusion, during infection, a pathogen perturbs the cell in a number of direct (process of invasion) and indirect (metabolic changes) ways which, in addition to detecting PAMPs, may be used to discriminate a pathogen from a harmless commensal.

\section{No Place to Hide: Activation of Innate Immune Responses}

Activation of immune responses is induced mainly by: (i) changes due to the presence of activators (i.e., PAMPs), which can be sensed by foreign versus self-discrimination; (ii) changes in spatial availability of activators (DAMP sensing); or (iii) pathogen-induced signaling changes within the cellular system. These temporal changes within the cell or its environment, all fit the requirements of the discontinuity theory of immunity [4]. Therefore, substances recognized by the innate immune receptor repertoire are not limited to classical structural PAMPs (such as LPS, flagellin, and peptidoglycan), but also include bacterial metabolites.

\section{Sensing of Bacterial Metabolites}

Quorum-sensing molecules, which are used by bacteria for inter- and intraspecies communication, represent one such example of recognition. The Pseudomonas aeruginosa quorum-sensing molecule 3-oxo-dodecanoyl-L-homoserine lactone, can be sensed by human host cells and enhance inflammatory responses, but can also dampen PRR-mediated responses for the benefit of the bacterium [21]. Vibrio vulnificus derived cyclo(Phe-Pro) can interfere with retinoic acid inducible gene (RIG)-I activation in human cells and in mice [22]. It remains to be established, however, whether quorum-sensing molecules are ubiquitously used to modulate and alarm the immune system.

Some bacterial metabolites that are structurally different from host metabolites (such as short-lived vitamin B synthesis intermediates), are bona fide targets for immune detection. A good example of this are MHC-I-related (MR1)-restricted mucosal-associated invariant $\mathrm{T}$ (MAIT) cells, which can be activated by short-lived bacterial vitamin B metabolites when the cells are proximal to metabolically active bacteria [23]. The tumor necrosis factor receptor (TNFR)-associated factor (TRAF)2 and TRAF6 binding protein TRAF-interacting protein with a forkhead-associated domain (TIFA) can induce oligomerization and activation of TRAF2/6, leading to downstream activation of the inhibitor of nuclear factor (NF)-KB kinase (IKK) complex [24]. One study showed that heat-resistant compounds derived from Neisseria spp. could act
Pathogen-associated molecular pattern (PAMP): molecule from pathogens that is sensed by host receptors (PRRs).

Patterns-of-pathogenesis hypothesis: theoretical framework of key features of pathogens that lead to sensing and induction of immune reactions and allow for discrimination from nonpathogenic microbes.

Peptidoglycan: polymer consisting of carbohydrates, crosslinked by amino acids that builds the bacterial cell wall. Pattern-recognition receptor (PRR) or PAMP receptor: receptor that senses PAMPs.

PAMP-triggered immunity (PTI): immune cascade in plants triggered by a PAMP.

Pyrin inflammasome: inflammasome complex composed of ASC, caspase-1, and pyrin.

Quorum-sensing molecules: small molecules used by bacteria for intra- and inter-species communication.

Retinoic acid inducible gene (RIG)-I: a cytosolic RNA sensor.

Unfolded protein response (UPR): cellular response in the ER to accumulated unfolded proteins and/or cellular stress.

Vita-PAMPs: PAMPs present only in living microorganisms. 
as activators of TIFA, inducing oligomerization and phosphorylation of the latter, thus resulting in IKK activation [25]. By genetically targeting enzymes of the bacterial ADP heptose pathway, the authors of this study initially proposed that the bacteria-derived monosaccharide beta heptose-1,7-bisphosphate (BHBP) was the activating compound [25]. However, ADP-heptose, was later identified as the active compound, with $\beta \mathrm{HBP}$ only being an intermediate requiring processing to ADP-heptose by host adenylytransferases $[26,27]$. Other studies focused on oligomerization of TIFA, shown to be phosphorylated by a protein kinase (ALPK)1 at threonine 9 [28,29]. Indeed, ALPK1 is the physiologically relevant receptor that directly binds ADP-heptose via its N-terminal domain [27]. In turn, ALPK1/TIFA-mediated detection of bacterial metabolic activity acts in a complementary manner to the NOD1/2 intracellular surveillance pathway, which subsequently detects the cytosolic presence of bacterial peptidogylcan structures (for review on NOD1/2, see [30]). The enteroinvasive bacterial pathogen Shigella spp. induces NOD1 activation at early stages of cytoinvasion, whereas TIFA is activated at later times of infection [31]. This redundancy makes it harder for bacteria to escape immunosurveillance, given that two essential bacterial molecules would need to be modified to achieve subversion [31]. The physiological contribution of TIFA to the immune detection of bacteria, however, is not entirely clear. Questions remain regarding the expression and contribution of the TIFA pathway to pathogen sensing in different cell types and tissues, and why it is not activated by commensal metabolites.

The use of host enzymes to generate active ligands of PRRs is probably a generalized mechanism in innate immunity, also exemplified by the sensing of cytosolic DNA - a universal danger signal sensed by the innate immune system [32]. This includes the sensing of pathogens, but also endogenous host DNAs [33]. DNA sensing involves several host proteins, among them, cyclic GMP-AMP synthase (cGAS). Direct recognition of DNA through cGAS is mediated by binding to the sugar-phosphate backbone [34,35], which induces type I interferon (IFN-I)-dependent antiviral and bacterial responses (for recent reviews see [33,36]). Several bacterial species produce cyclic dinucleotides as secondary messengers affecting numerous responses of the prokaryote. In mammalian hosts, these cyclic dinucleotides can activate the cGAS/STING pathway. Specifically, cGAS was shown to contribute to innate immune sensing of Mycobacterium tuberculosis [37-39], Francisella tulariensis [40,41], Legionella pneumophila, and Chlamydia trachomatis [39,42] as well as Listeria monocytogenes [43]. There are differences in how Listeria is detected in mice and humans, given that the IFN response depends on CGAS and STING in human THP1 cells, but is cGAS-independent in mouse macrophages $[37,41]$. The dual use of the bacterial metabolite $\left(3^{\prime}-5^{\prime}, 3^{\prime}-5^{\prime} \mathrm{cGAMP}\right)$ and the endogenously produced cyclic dinucleotide with a mixed-linkage backbone, (2'-5', $3^{\prime}-5^{\prime} \mathrm{c}$ GAMP) are interesting features because mouse and human cGAS differentially detect these two cyclic dinucleotides [44] (Figure 1, Key Figure). In humans, STING is polymorphic and many alleles differ in their discrimination between endogenous and bacterial cyclic dinucleotides (i.e., 3'-5',3'-5'cGAMP vs 2'-5',3'-5'cGAMP). Polymorphisms in STING might thus have implications regarding susceptibility to infectious disease, as recently suggested for human Legionnaires' disease [45] (See Table 1).

Bacterial DNA is recognized by endosomal TLR9 [46], but it is clear that this receptor also recognizes host mtDNA, given that the latter contains unmethylated CpG DNA repeats that are similar to those within bacterial genomes [47]. TLR9 activation causes NF-KB activation and proinflammatory cytokine transcriptional changes, so its recognition of mtDNA may be associated with certain inflammatory diseases, as proposed for atherosclerosis [48]. Direct recognition of DNA through cGAS also occurs in response to endogenous DNAs, including mtDNA [49]. Activation of the cGAS-STING pathway by leakage of mtDNA into the cytosol has been implicated in the etiology of infectious and autoinflammatory disorders (e.g., systemic lupus erythematosus) [50]. Herpes simplex and dengue virus infections are thought to lead to mtDNA leakage and 


\section{Key Figure}

Mammalian Innate Immune Activation by Bacterial Pathogens

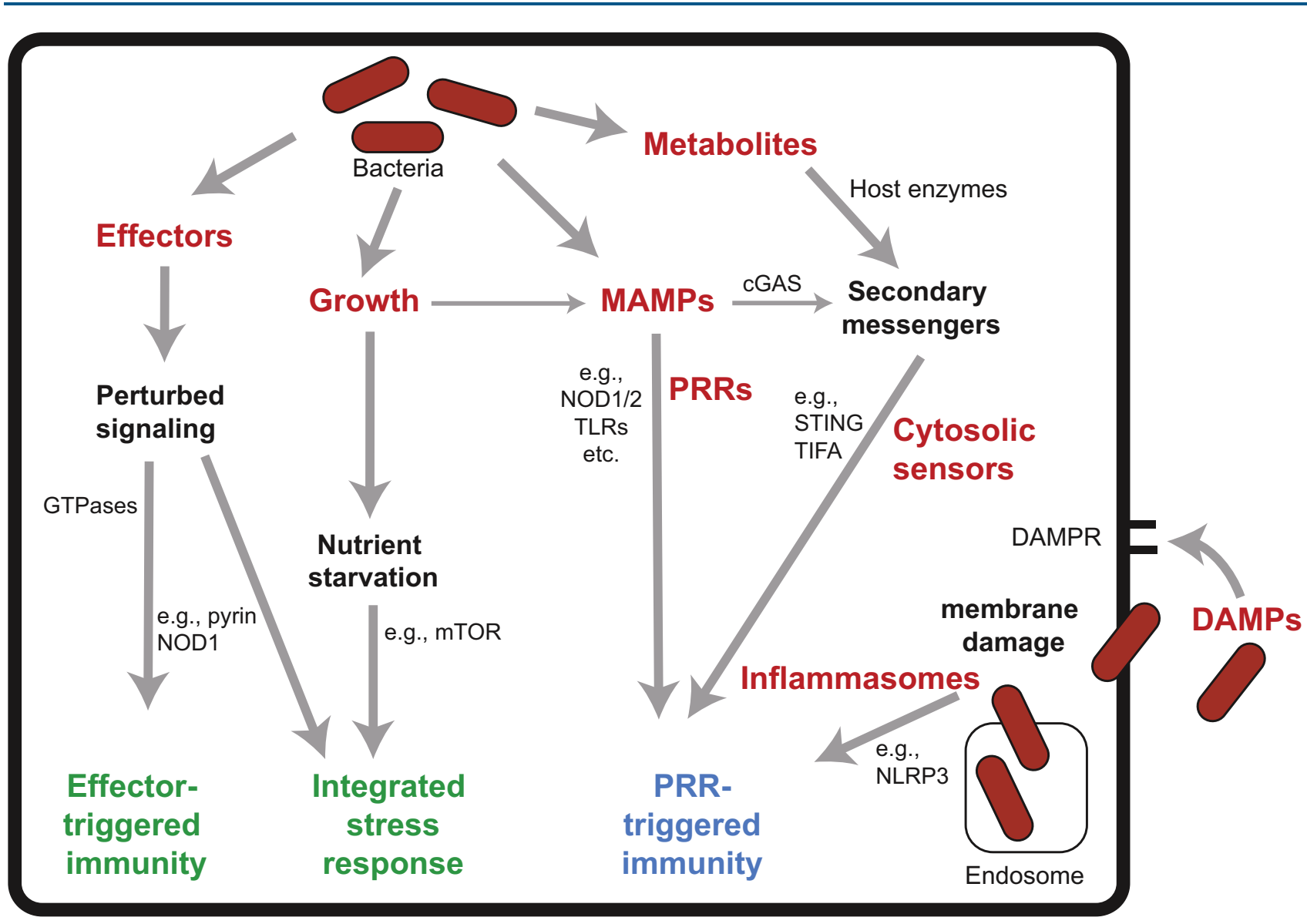

Cell membrane

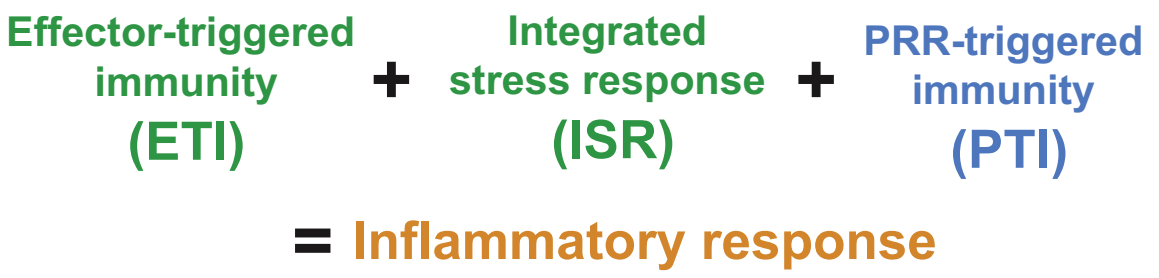

Trends in Immunology

Figure 1. Schematic overview of mammalian innate immune activation by bacterial pathogens and the interconnection of effector-triggered immunity (ETI), PAMPtriggered immunity (PTI), and integrated stress response (ISR) pathways, leading to proinflammatory responses and induction of immunity. Abbreviations: cGAS, cyclic GMP-AMP synthase; DAMP, damage-associated molecular pattern; DAMPR, DAMP receptor; MAMP, microbial-associated molecular pattern; mTOR, mammalian target of rapamycin; NLRP3, NLR family pyrin domain containing 3, NOD1/2, nucleotide-binding oligomerization domain-containing protein 1/2; PAMP, pathogenactivated molecular pattern; STING, stimulator of interferon genes; TIFA, TRAF-interacting protein with a forkhead-associated domain; TLR, Toll-like receptor.

insufficiency of host DNA-binding proteins, such as TFAM, that promote cGAS DNA recognition [51]. Bacteria-derived metabolites and signaling molecules can thus be seen as a type of PAMP which is recognized, directly or indirectly, by the host cell. 
Table 1. Examples of PRRs and Their ETI and PTI Activators ${ }^{a}$

\begin{tabular}{|c|c|c|c|}
\hline Sensor (PRR) & PTI ligand/activator & ETI ligand/signal & Signaling pathway \\
\hline NOD1/2 & Peptidogylcan & RhoGTPase, F-actin & NF-kB, MAPK \\
\hline NLRPS & DAMPs & MT, F-actin & Inflammasomes \\
\hline NLRP1 & $\begin{array}{l}\text { MDP (?), Toxoplasma } \\
\text { gondii }\end{array}$ & $\begin{array}{l}\text { Functional degradation, } \\
\mathrm{LF}, \mathrm{IpaH7} .8\end{array}$ & Inflammasomes \\
\hline NLRC4/NAIP & T3SS & Unknown & $\begin{array}{l}\text { Inflammasomes, F-actin } \\
\text { polymerization }\end{array}$ \\
\hline TLR4, 2, 6 & Lipoproteins, LPS & Unknown & NF-kB, MAPK \\
\hline TLR3, 7, 8, 9 & Nucleic acids & Unknown & NF-KB, MAPK \\
\hline cGAS/STING & 3'-5', 3'-5'cGAMP & 2'-5', 3'-5'cGAMP & Type I IFN \\
\hline RIG-I & Nucleic acids & Unknown & Type I IFN \\
\hline Pyrin & Unknown & PKN1/2 inactivation & Inflammasomes \\
\hline ALPK1/TIFA & ADP-heptose & Unknown & $N F-k B$ \\
\hline
\end{tabular}

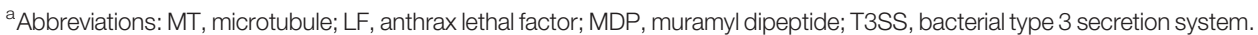

Sensing of Cellular Stress

Other changes in the cell sensed by innate receptors are membrane damage; for example, some enteroinvasive bacteria can induce depletion of amino acids by membrane damage, resulting in the activation of mammalian target of rapamycin (mTOR)-controlled autophagy or the integrated stress response via the general control non-de-repressible 2 kinase (GCN2, EIF2AK4), as demonstrated in Shigella-infected human cell lines [52]. Many bacteria, including Brucella abortus and Chlamydia muridarum, are known to induce endoplasmic reticulum (ER) stress responses, resulting in activation of the unfolded protein response (UPR) effectors, inositol-requiring enzyme (IRE)1 a, ATF6, and PRKR-like endoplasmic reticulum kinase (PERK) (Figure 1) [53]. Upon mouse infection with Brucella and Chlamydia, UPR can ultimately lead to the activation of inflammatory pathways by an IREa-mediated TRAF2-NOD1/2 pathway that is independent of NOD1/2-mediated peptidoglycan sensing [54]. In analogy to the ETI response in plants, where immunity is induced by sensing of bacterial effector-mediated degradation or modification of cytosolic host proteins, evidence for direct activation of mammalian PRRs by bacterial enzymes is emerging. Recently, mouse NLRP1b - an inflammasome-forming NLR protein - was shown to be activated by the cleavage by Bacillus anthrax lethal factor (LF) and by ubiquitination via the Shigella ssp. IpaH7.8 protein, which directs proteasomal N-terminal NLRP1b degradation in cell lines $[55,56]$.

At least some cytosolic PRRs are not bona fide cytosolic proteins, but rather, show defined subcellular localization patterns that may be altered upon activation. In mouse embryonic fibroblasts and human cell lines, for example, activated STING moves from the ER to become enriched at perinuclear compartments [57]. Localization of STING at the mitochondria plays central roles in orchestrating innate immune signaling. Mitochondrial-associated membranes (MAMs) - the ER membranes juxtaposed to mitochondria - also provide a scaffolding role in IFN signaling, as they constitute a site of STING localization [58]. cGAS is located at the cell membrane and membrane localization is important for adequate sensing function [59]. Active NOD1 and NOD2 are localized at F-actin-rich regions at the cell membrane [60-62] and NOD1-mediated activation of the downstream receptor-interacting serine/threonine-protein kinase (RIPK)2 can occur at early endosomes in cell lines [63]. This defined localization seems to coincide with their role as sensors, allowing a sensitive response to PAMPs that are released from invading or endosomal bacteria, and the detection of mislocalized (i.e., cytosolic) DNA (in the case cGAS). 
Mitochondria: Emerging Integrators of Immune Sensing

Of all the subcellular organelles, mitochondria are emerging as some of the most important platforms for activation of several innate immune signaling pathways, including inflammasome activation. Many activators of the NLRP3 inflammasome disrupt mitochondrial integrity which generates reactive oxygen species (ROS) and releases, among other things, mtDNA into the cytosol [64,65]. Exposure of the inner mitochondrial membrane lipid cardiolipin can contribute to both NLRP3 activation as well as ROS production by enabling the formation of the inflammasome complex in cell lines [66]. Moreover, the release of oxidized mtDNA from damaged mitochondria into the cytosol can stimulate NLRP3 to drive interleukin (IL)-1 $1 \beta$ production [67]. Relative to controls, LPS activation of TLR4 induces an IFN regulatory factor (IRF)1- and mitochondrial cytidine/uridine monophosphate kinase (CMPK)2-dependent increase in mtDNA synthesis and release of oxidized mtDNA into the cytoplasm of mouse BMDMs, potentially linking mitochondrial function to the priming of NLRP3 activity [68]. In humans and mice, cytosolic DNA can also be sensed by another inflammasome forming receptor, absent in melanoma (AIM)2 [69,70]. It is reasonable to assume that AIM2 could also sense mtDNA, although to date, most of the data suggests receptor crosstalk between mtDNA and the AIM2 inflammasome receptor, rather than direct binding of mtDNA to this receptor [71]; this makes AIM2 a likely candidate for being a direct mtDNA receptor. Mitochondria can also regulate the balance between apoptosis and inflammation: when apoptosis is switched on, inflammatory signals are switched off, and vice versa. This is subverted by intracellular bacterial pathogens such as Shigella spp. [119]. Consequently, mitochondria and their associated metabolites may constitute a central platform coordinating a number of innate immune signaling responses (for a review on the role of mitochondria in innate immunity, see [72]).

\section{Skeleton Crew: Contribution of the Cytoskeleton to Innate Immunity}

The cytoskeleton is composed of different dynamic filament-forming proteins including actin, microtubules, intermediate filaments, and septins. Among the most important and best understood in the context of bacterial infection are F-actin and microtubules (Box 1) [73]. It is becoming clear that cytoskeletal dynamics are often essential for both the detection of microbial components within cells and the initiation of appropriate cellular innate immune responses. The importance of cytoskeletal dynamics during host defense is highlighted by the abundance of bacterial toxins/virulence factors that specifically target the ability of the host cell to alter its cytoskeleton, allowing the pathogen to survive (Box 2).

\section{Actin and Microtubules}

The dense actin cortex, found directly beneath the plasma membrane, provides rigidity to the cell, acting as a scaffold to control the localization of receptors and other proteins, enabling the

\section{Box 1. Cytoskeletal Structures}

\section{Actin}

Actin filaments (F-actin) are polar dynamic structures generated by the ATP-dependent polymerization of actin monomers (G-actin). Actin filament disassembly occurs to replenish the pool of monomeric actin, from which new filaments can be generated, or existing filaments can be elongated. A significant pool of actin subunits are thus constantly in flux between monomeric and filamentous states. Rapid dynamics of the intracellular actin cytoskeleton are required so that it can perform its functional activities within the cell, that are essential for a range of innate immune responses, including changes in cell shape, rapid cell motility, phagocytosis, transepithelial migration, signal transduction, and transcriptional regulation [109].

\section{Microtubules}

Microtubules are hollow cylinders composed of $\alpha$ - and $\beta$-tubulin subunits that form GTP-bound dimers that can arrange to long polymers. Microtubule filaments are involved in the intracellular compartmentalization of cellular structures and organelles, and serve as tracks for the intracellular transport of proteins/organelles. Microtubule polymerization is highly dynamic and is regulated by several proteins. Post-translational modification (PTM) is a key event and acetylation of lysine 40 in $\alpha$-tubulin is found in stable microtubules, which can be edited by SIRT2, and other enzymes [110]. 


\section{Box 2. Examples of Bacterial-Induced Actin Modifications}

Further evidence for actin polymerization being an essential factor for host defense includes the abundance of pathogenic factors that specifically target the host's ability to polymerize actin, so as to gain a survival advantage within the host [111]. Toxins from numerous different bacterial species modify actin, via ADP ribosylation, to impair its normal functional activity. Specifically, toxins from bacteria including Clostridium botulinum (C2), Clostridium difficile (CDT), Salmonella enterica (SpvB), and Aeromonas salmonicida (AexT) all inhibit actin polymerization by ADP-ribosylation of G-actin at Arg177 [112]. In contrast, ADP ribosylation of actin at Thr148 by the Photorhabdus luminescens toxin (TccC3) induces aggregation and bundling of cellular actin filaments in human HeLa cells, which are largely resistant to depolymerization [113]. Another family of toxins, which are present in species including Vibrio cholerae and Aeromonas hydrophila, are capable of crosslinking actin monomers into dimers, trimers, and higher order oligomers that are incapable of incorporation into filaments, and furthermore, serve to sequester and potently inhibit many actin assembly factors, including the ARP2/3 complex, Spire, and Formins [114]. Alternative strategies to PTM are also used by bacteria to target the host's actin cytoskeleton, including the direct processing of actin by bacterial metalloproteases, such as ECP32 from Serratia proteamaculans and RavK from Legionella pneumophila which directly cleave actin, abolishing its ability to be incorporated into polymers in human epithelial cell lines and COS-1 cells [115,116].

Strategies to target regulators of the actin host machinery, such as the Rho proteins, are also widely used by bacterial factors. Rho family small GTPases are critical signaling adaptors that interpret extracellular stimuli and orchestrate a wide range of cellular responses, including dynamic regulation of the actin cytoskeleton. PTMs including ADP ribosylation, adenylylation, proteolysis, and deamidation are used by bacterial species to inhibit Rho function [112]. SET domain protein (SETD)3 has recently been identified as the enzyme responsible for the methylation of actin at His73 [117]. Many actin isoforms are methylated at His73; a residue localized in close proximity to the actin nucleotide binding site. The ability of SETD3-mediated methylation of actin to modulate actin dynamics has been demonstrated by experiments showing that purified nonmethylated actin exhibits slower polymerization rates than methylated actin, and SETD3-deficient mouse embryonic fibroblasts have slower migration rates than their WT counterparts. We therefore speculate that SETD3 is likely to be uncovered as an additional target of bacterial species with an ability to modify actin dynamics within host cells.

endocytosis of receptors, such as TLRs [74]. Actin rearrangement can also change the susceptibility of cells to infection and regulate bacterial induced changes in inflammasome function [75]. Finally, microbe induced alterations in actin dynamics and in actin-modulating proteins can be sensed by the cell, leading to the concept of the mammalian guard hypothesis.

Phagocytosis is a specialized form of endocytosis in which large particles such as pathogens, dead cells, and other particles are recognized via specific PRRs, FcR, or complement receptors. These distinct mechanisms of phagocytosis all require dynamic regulation of the actin cytoskeleton [76]. Once internalized, the phagosome vacuoles fuse selectively with primary lysosomes, or the product of the ER and Golgi complex, to form a secondary phagolysosome. Cellular responses to phagocytosis vary depending on cell type, the receptors involved, and the nature of the cargo. In macrophages, vacuole contents are subjected to progressive acidification and digestion by proteolytic enzymes and lysosomal DNase II [77,78]. The presence of the liberated DNA in the cytosol following digestion can induce proinflammatory cytokine production via the cGAS-STING pathway, as demonstrated in DNA transfection and DNA viral infection experiments of human THP1 monocytic cells and murine L929 fibroblasts [34,79]. Seminal studies have demonstrated that incomplete proteolysis occurs in dendritic cells (DCs) to facilitate $\mathrm{MHC}-1 / \mathrm{II}$-mediated antigenic peptide presentation to $\mathrm{CD}^{+} / \mathrm{CD} 8^{+} \mathrm{T}$ cells $[80,81]$. In neutrophils and other granulocytes, degranulation occurs in response to phagocytosis, causing the extracellular release of antimicrobial granule contents [76].

TLRs are not capable of inducing phagocytosis in isolation, but they do cooperate with phagocytic receptors to modulate the cellular response, and also promote the uptake of soluble antigens by endocytosis [74]. For example, coactivation of TLR2 and the phagocytic $\beta$-glucan receptor, dectin-1, results in synergistic production of TNF- $\alpha$ in murine RAW264.7 macrophages and bone marrow-derived DCs [82,83]. Indeed, the actin cytoskeleton appears to be essential for the endocytosis of these receptors. Moreover, TLR4-mediated activation of NF-KB and MAPK 
signaling pathways have been shown to be mediated from the plasma membrane, but actinmediated endocytosis of TLR4 is required for IFN-I signaling in mouse BMDM and cell lines [84]. Similarly, actin-dependent maturation of TLR9 endosomes can control expression of type I IFNs in mouse DCs and macrophages [85].

A number of studies provide evidence of a host response to bacteria-induced alterations in the cytoskeleton (Box 2), supporting the mammalian guard hypothesis (Figure 1). For example, Salmonella-induced activation of NLRC4 in murine BMDMs has been shown to drive actin rearrangement, which prevents further cellular infection from occurring [86]. Exposure of cardiolipin at bacterial cell division fission sites leads to recruitment of host septins in cell lines [17]. NOD1, a cellular sensor for bacterial peptidoglycan, can be activated by small GTPases [87] and actin remodeling has been linked to enhanced NOD1- and NOD2-mediated proinflammatory signaling in human epithelial cells [88-91]. The pyrin inflammasome is indirectly activated following infection with bacteria such as Clostridium difficile and Burkholderia cenocepacia, which utilize toxins to inactivate Rho GTPase and induce actin depolymerization [92]. Specifically, wild-type (WT) mouse BMDMs treated with the secreted C. difficile cytotoxin TcdB, or infected with B. cenocepacia, induced associated speck-like protein containing a CARD (ASC) inflammasome specks, indicative of inflammasome activation. Furthermore, overexpressed RhoA from B. cenocepacia infected DC2.4 cells was purified and analyzed by mass spectrometry, identifying Asn 41 as the specific deamidation site on RhoA that was responsible for pyrin inflammasome activation [92]. Although not an NLR, pyrin (encoded by MEFV) has an N-terminal pyrin homology domain (PYD) that enables homotypic interactions, such that it oligomerizes with ASC and recruits caspase- 1 to form active inflammasome structures [92]. Two RhoA effector kinases, protein kinase (PK)N1 and PKN2 phosphorylate pyrin at Ser 208 and Ser 242, allowing for 14-3-3 binding; this results in the inhibition of the pyrin inflammasome in the presence of functional RhoA in murine BMDMs [93]. The presence of a priming step and RhoA inactivation abolishes the normal cellular restrictions on pyrin inflammasome activity, thereby enabling IL-1 $\beta$-mediated host defense against bacteria via detection of specific virulence-related activities, rather than via direct PAMP detection [93]. Familial Mediterranean fever (FMF) is an autoinflammatory disease caused by MEFV mutations that are likely to block the phosphorylation sites from PKN1 and PKN2, resulting in a lower activation threshold for the mutated pyrin inflammasome compared to the common allele [93]. FMF has long been effectively managed in patients with lifelong administration of the microtubule destabilizing drug colchicine, [94], suggesting that microtubules contribute to the regulation of the pyrin inflammasome. Depolymerization of microtubules by colchicine causes the release of the RhoA activator, guanine nucleotide exchange factor (GEF)-H1; in vitro data has shown that colchicine treatment (>100 ng/ml) activates RhoA in LPS-primed mouse BMDMs, and reverses clostridial C3 toxin mediated-inhibition of RhoA activity, with subsequent inhibition of the pyrin inflammasome [93]. These studies highlight the importance of RhoA and cytoskeletal dynamics as central integrators of pathogen-induced perturbations. F-actin from different species has also been identified as a DAMP on dead cells, binding the C-type lectin receptor CLEC9A to activate DCs $[95,96]$, and suggesting that actin can act as a universal hub of immune sensing in mammalian cells.

Compounds, such as nocodazole and colchicine, that disorganize microtubule organization, reduce NLRP3 inflammasome activation in LPS-primed, nigericin-stimulated murine macrophage cells [97]. NLRP3 localizes to ER structures, but upon activation, NLRP3 and its adaptor ASC, redistribute to the ER and the mitochondria [64] in a microtubule-dependent manner that involves the regulation of tubulin acetylation in BMDMs $[98,99]$. Tubulin acetylation can be also be induced by the acyltransferase MEC-17 and counteracted by the enzyme sirtuin (SIRT)2, leading to decreased or enhanced NLRP3 activation upon targeting of these enzymes, respectively [98]. 
Taken together, these studies show that other constituents of the cellular cytoskeleton, along with actin, can contribute to the regulation and induction of innate immune responses.

\section{Guardians of the Galaxy: Pathogen Recognition across Phyla, the Guard Hypothesis, and Beyond}

Unlike animals, plants rely solely on innate immunity for protection against pathogens. Much of the work on plant immunity predates our understanding of innate immunity in animals. The plant innate immune system is composed of extracellular PRRs and intracellular NLRs that recognize bacterial effectors delivered into host cells [100]. ETI in plants, as proposed by the guard hypothesis is, therefore, a key area of similarity between plant and animal disease resistance $[101,102]$. Resistance in plants against infections can be local (for example, alteration in cell wall defense), basal (reduced pathogen spread from the inoculation site following successful infection), or systemic (distal to the inoculation site, conferring broad spectrum resistance against pathogens) [100]. Local resistance, race-specific resistance (vertical resistance), or ETI involve some form of pathogen recognition and/or association either directly or indirectly, with plant resistant proteins [100]. These mechanisms of local and basal plant immunity are rather well understood. Plants do not, however, have a comparable adaptive immune system to animals, and the possibility of immune memory has not been thought to occur.

Innate immunity in animals was originally thought to be devoid of a memory component, but it is increasingly clear that this is not the case [103]. Macrophages, for example, have long been known to have plasticity such that following first exposure to a PAMP, PRR expression can change, and a subsequent challenge with a broad spectrum of pathogens leads to increased innate immune activity $[104,105]$. Depending on the initial dose of LPS (e.g., in macrophages), the cell can undergo priming (enhancement) or tolerance (suppression) upon a secondary challenge with LPS, representing a well-known example of this type of innate immune memory [106]. Moreover, macrophages or monocytes stimulated initially with Candida albicans or $\beta$-glucans and receiving a secondary challenge with an unrelated pathogen or PAMP show enhanced inflammatory responses relative to a primary challenge, processes thought to be regulated via epigenetic reprogramming [103]. The mechanistic basis for this might involve myeloid skewing of the hematopoietic compartment and cell-intrinsic changes (including changes in glycolysis, mevalonate synthesis, and mTOR activation); this has led to the concept of innate immune memory or trained immunity [103].

Plants generate a systemic-acquired resistance (SAR) triggered by infection that provides a longlasting and broad-spectrum protection against related and unrelated pathogens [107]. It requires a barrier (the waxy cuticle) and a set of diverse chemical SAR inducers including plant hormones, metabolites and proteins [108]. A mobile signal generated at the site of infection travels elsewhere in the plant to confer resistance against future infections which requires a complex interplay between phytohormones, metabolites, and proteins. Similarly to trained immunity, SAR involves epigenetic changes including patterns of DNA methylation at the promoters of defense-related genes and these can be transmitted to the next generation of progeny [107]. The concept of a memory immunity in plants provides remarkable potential opportunities for breeding diseaseresistant crops. It is now increasingly clear that an equivalent to trained immunity or SAR exists across the animal and plant kingdoms including microbes; precisely how this type of immunity can be beneficially translated is an exciting concept for future research.

\section{Concluding Remarks}

In summary, induction of innate immune responses by pathogens relies mainly on direct sensing of pathogen-derived molecules. Amplification of these by host enzymes provides a further means to
Outstanding Questions

Are there main hubs that link detection of cellular perturbations to innate immune signaling?

Perturbation of small GTPases is common to many mammalian ETI responses, raising the question that this may be a key signature - or it may just reflect a current lack of knowledge on other mechanisms.

What are the mechanisms of detection of pathogen-induced cytoskeletal perturbations?

NLR proteins and pyrin have been implicated in sensing pathogen-induced cytoskeletal perturbations; however, the molecular mechanisms have only been identified for a few particular cases.

Do other triggers for ETI exist and what are they? Research on ETI in mammals is based on similarities to plant immunity, however whether mammalianspecific triggers for ETI exist is still unclear.

What is the physiological role of mammalian ETI in the induction of adaptive immunity?

PRR activation is a prerequisite to induce adaptive immunity; however, it is still unclear if ETI is sufficient to trigger adaptive responses in the absence of PRR activation. 
generate robust antipathogenic responses. The sensing of host cytoskeleton perturbations and pathogen interference with these signaling cascades - collectively described as ETI - enhance innate immune responses. The current literature suggests that ETI alone might not be able to induce sufficiently strong responses against infections with bacterial pathogens. Indeed, in most cases, in addition to the ETI trigger, priming of myeloid cells or simultaneous activation of several classical PRR pathways is required to induce the transcription of the full cohort of effector molecules to generate activation of innate immunity (e.g., as in the case of the pyrin inflammasome). This suggests that mammalian ETI, in contrast to plant ETI, might serve as an amplification mechanism to induce robust innate immune responses, but might not be sufficient to lead to inflammatory responses without co-stimulation by PAMPs (Figure 1). The detrimental consequences of inappropriate activation of innate and downstream adaptive immune responses in animals make it tempting to speculate that this system might have evolved as a safeguard to avoid tissue damage and autoimmune reactions. Lastly, we propose that robust, pathogen-induced cell-autonomous immunity in mammalian cells utilizes both the activation of PRRs accompanied by amplification of these responses by simultaneous sensing of bacterial perturbation of the cytoskeleton, cell metabolism, or cellular signaling. Extensive work is required to explore this hypothesis and to determine its putative underlying molecular mechanisms (see Outstanding Questions).

\section{Acknowledgments}

Limitations on the number of references precluded us from citing many important contributions, and we appreciate the understanding of colleagues whose work could not be cited. C.E.B. is supported by a Wellcome Trust Investigator Award (108045/Z/15/Z). E.M.C. acknowledges funding from the Irish Research Council (IRC, GOIPG/2018/3407) and Science Foundation Ireland (SFI, 16/TIDA/4004). T.A.K. acknowledges support by the German Research Foundation (DFG), grant KU 1945/4-1.

\section{References}

1. Medzhitov, R. (2009) Approaching the asymptote: 20 years later. Immunity 30, 766-775

2. Matzinger, P. (1994) Tolerance, danger, and the extended family. Annu. Rev. Immunol. 12, 991-1045

3. Bianchi, M.E. (2007) DAMPs, PAMPs and alarmins: all we need to know about danger. J. Leukoc. Biol. 81, 1-5

4. Pradeu, T. et al. (2013) The speed of change: towards a discontinuity theory of immunity? Nat. Rev. Immunol. 13, 764-769

5. Vance, R.E. et al. (2009) Patterns of pathogenesis: discrimination of pathogenic and nonpathogenic microbes by the innate immune system. Cell Host Microbe 6, 10-21

6. Girardin, S.E. et al. (2003) Nod1 detects a unique muropeptide from gram-negative bacterial peptidoglycan. Science 300 , 1584-1587

7. Ogura, Y. et al. (2001) A frameshift mutation in NOD2 associated with susceptibility to Crohn's disease. Nature 411 , 603-606

8. Shi, J. et al. (2014) Inflammatory caspases are innate immune receptors for intracellular LPS. Nature 514, 187-192

9. Ruter, C. et al. (2018) All in-multiple parallel strategies for intracellular delivery by bacterial pathogens. Int. J. Med. Microbiol. 308, 872-881

10. Rathinam, V.A. and Fitzgerald, K.A. (2011) Cytosolic surveillance and antiviral immunity. Curr. Opin. Virol. 1, 455-462

11. Ugolini, M. and Sander, L.E. (2018) Dead or alive: how the immune system detects microbial viability. Curr. Opin. Immunol. 56, 60-66

12. Blander, J.M. and Sander, L.E. (2012) Beyond pattern recognition: five immune checkpoints for scaling the microbial threat. Nat. Rev. Immunol. 12, 215-225

13. Barbet, G. et al. (2018) Sensing microbial viability through bacterial RNA augments T follicular helper cell and antibody responses. Immunity 48, 584-598.e5

14. Sander, L.E. et al. (2011) Detection of prokaryotic mRNA signifies microbial viability and promotes immunity. Nature 474, 385-389
15. Ugolini, M. et al. (2018) Recognition of microbial viability via TLR8 drives TFH cell differentiation and vaccine responses. Nat. Immunol. 19, 386-396

16. Moretti, J. et al. (2017) STING senses microbial viability to orchestrate stress-mediated autophagy of the endoplasmic reticulum. Cell 171, 809-823.e13

17. Krokowski, S. et al. (2018) Septins recognize and entrap dividing bacterial cells for delivery to lysosomes. Cell Host Microbe 24, 866-874.e4

18. Brito, C. et al. (2019) Mechanisms protecting host cells against bacterial pore-forming toxins. Cell. Mol. Life Sci. 76 , 1319-1339

19. Perez-Lopez, A. et al. (2016) Mucosal immunity to pathogenic intestinal bacteria. Nat. Rev. Immunol. 16, 135-148

20. Vaishnava, S. et al. (2011) The antibacterial lectin Reglllgamma promotes the spatial segregation of microbiota and host in the intestine. Science 334, 255-258

21. Glucksam-Galnoy, Y. et al. (2013) The bacterial quorumsensing signal molecule N-3-oxo-dodecanoyl-L-homoserine actone reciprocally modulates pro- and anti-inflammatory cytokines in activated macrophages. J. Immunol. 191, 337-344

22. Lee, W. et al. (2018) Vibrio vulnificus quorum-sensing molecule cyclo(Phe-Pro) inhibits RIG-I-mediated antiviral innate immunity. Nat. Commun. 9, 1606

23. McWilliam, H.E. and Villadangos, J.A. (2018) MR1 antigen presentation to MAIT cells: new ligands, diverse pathways? Curr. Opin. Immunol. 52, 108-113

24. Ea, C.K. et al. (2004) TIFA activates IkappaB kinase (IKK) by promoting oligomerization and ubiquitination of TRAF6. Proc Natl. Acad. Sci. U. S. A. 101, 15318-15323

25. Gaudet, R.G. et al. (2015) Innate immunity. Cytosolic detection of the bacterial metabolite HBP activates TIFA-dependent innate immunity. Science 348, 1251-1255

26. Garcia-Weber, D. et al, (2018) ADP-heptose is a newly identified pathogen-associated molecular pattern of Shigella flexneri. EMBO Rep. 19 
27. Zhou, P. et al. (2018) Alpha-kinase 1 is a cytosolic innate immune receptor for bacterial ADP-heptose. Nature 561, 122-126

28. Milivojevic, M. et al. (2017) ALPK1 controls TIFA/TRAF6dependent innate immunity against heptose-1,7-bisphosphate of gram-negative bacteria. PLoS Pathog. 13, e1006224

29. Zimmermann, S. et al. (2017) ALPK1- and TIFA-dependent in nate immune response triggered by the Helicobacter pylori type IV secretion system. Cell Rep. 20, 2384-2395

30. Philpott, D.J. et al. (2014) NOD proteins: regulators of inflammation in health and disease. Nat. Rev. Immunol. 14, 9-23

31. Gaudet, R.G. et al. (2017) Innate recognition of intracellula bacterial growth is driven by the TIFA-dependent cytosolic surveillance pathway. Cell Rep. 19, 1418-1430

32. Dhanwani, R. et al. (2018) Cytosolic sensing of immunostimulatory DNA, the enemy within. Curr. Opin. Immunol. 50, 82-87

33. Cai, X. et al. (2014) The cGAS-cGAMP-STING pathway of cytosolic DNA sensing and signaling. Mol. Cell 54, 289-296

34. Sun, L. et al. (2013) Cyclic GMP-AMP synthase is a cytosolic DNA sensor that activates the type I interferon pathway. Science 339, 786-791

35. Civril, F. et al. (2013) Structural mechanism of cytosolic DNA sensing by cGAS. Nature 498, 332-337

36. Ablasser, A. and Gulen, M.F. (2016) The role of cGAS in innate immunity and beyond. J. Mol. Med. (Berl.) 94, 1085-1093

37. Collins, A.C. et al. (2015) Cyclic GMP-AMP synthase is an innate immune DNA sensor for Mycobacterium tuberculosis. Cell Host Microbe 17, 820-828

38. Wassermann, R. et al. (2015) Mycobacterium tuberculosis differentially activates cGAS- and inflammasome-dependent in tracellular immune responses through ESX-1. Cell Host Microbe 17, 799-810

39. Watson, R.O et al. (2015) The cytosolic sensor cGAS Detects Mycobacterium tuberculosis DNA to induce type I interferons and activate autophagy. Cell Host Microbe 17, 811-819

40. Man, S.M. et al. (2015) The transcription factor IRF1 and quanylate-binding proteins target activation of the AIM2 inflammasome by Francisella infection. Nat. Immunol. 16 467-475

41. Storek, K.M. et al. (2015) cGAS and Ifi204 cooperate to produce type I IFNs in response to Francisella infection. J. Immunol. 194, 3236-3245

42. Zhang, $Y$, et al (2014) The DNA sensor, cyclic GMP-AMP synthase, is essential for induction of IFN-beta during Chlamydia trachomatis infection. J. Immunol. 193, 2394-2404

43. Hansen, K. et al. (2014) Listeria monocytogenes induces IFNbeta expression through an IFI16-, cGAS- and STING dependent pathway. EMBO J. 33, 1654-1666

44. Danilchanka, O. and Mekalanos, J.J. (2013) Cyclic dinucleotides and the innate immune response. Cell 154, 962-970

45. Ruiz-Moreno, J.S, et al. (2018) The common HAQ STING variant impairs cGAS-dependent antibacterial responses and is associated with susceptibility to Legionnaires' disease in humans. PLoS Pathog. 14, e1006829

46. Hemmi, H. et al. (2000) A Toll-like receptor recognizes bacterial DNA. Nature 408, 740-745

47. Zhang, Q. et al. (2010) Circulating mitochondrial DAMPs cause inflammatory responses to injury. Nature 464, 104-107

48. Zhang, Z. et al. (2015) Mitochondrial DNA-LL-37 complex promotes atherosclerosis by escaping from autophagic recognition. Immunity 43, 1137-1147

49. West, A.P. et al. (2015) Mitochondrial DNA stress primes the antiviral innate immune response. Nature 520, 553-557

50. Gkirtzimanaki, K. et al. (2018) IFNalpha impairs autophagic degradation of mtDNA promoting autoreactivity of SLE monocytes in a STING-dependent fashion. Cell Rep. 25, 921-933.e5

51. Aguirre, S. et al. (2017) Dengue virus NS2B protein targets cGAS for degradation and prevents mitochondrial DNA sensing during infection. Nat. Microbiol. 2, 17037

52. Tattoli, I. et al. (2012) Amino acid starvation induced by invasive bacterial pathogens triggers an innate host defense program. Cell Host Microbe 11, 563-575

53. Rodrigues, L. et al. (2018) Integrated stress responses to bacterial pathogenesis patterns. Front. Immunol. 9, 1306
54. Keestra-Gounder, A.M. et al. (2016) NOD1 and NOD2 signalling links ER stress with inflammation. Nature 532, 394-397

55. Sandstrom, A. et al. (2019) Functional degradation: a mechanism of NLRP1 inflammasome activation by diverse pathogen enzymes. Science. Published online April 5, 2019. https://doi.org/10.1126/science.aau1330

56. Chui, A.J. et al. (2019) N-terminal degradation activates the NLRP1B inflammasome. Science 364, 82-85

57. Dobbs, N. et al. (2015) STING activation by translocation from the ER is associated with infection and autoinflammatory disease. Cell Host Microbe 18, 157-168

58. Missiroli, S. et al. (2018) Mitochondria-associated membranes (MAMs) and inflammation. Cell Death Dis. 9, 329

59. Barnett, K.C. et al. (2019) Phosphoinositide interactions position CGAS at the plasma membrane to ensure efficient distinction between self- and viral DNA. Cell 176, 1432-1446.e11

60. Kufer, T.A. et al. (2008) The pattern-recognition molecule Nod 1 is localized at the plasma membrane at sites of bacterial interaction. Cell. Microbiol. 10, 477-486

61. Lecine, P. et al. (2007) The NOD2-RICK complex signals from the plasma membrane. J. Biol. Chem. 15197-15207

62. Schaefer, A.K. et al. (2017) Membrane association dictates ligand specificity for the innate immune receptor NOD2. ACS Chem. Biol. 12, 2216-2224

63. Irving, A.T. et al. (2014) The immune receptor NOD1 and kinase RIP2 interact with bacterial peptidoglycan on early endosomes to promote autophagy and inflammatory signaling Cell Host Microbe 15, 623-635

64. Zhou, R. et al. (2011) A role for mitochondria in NLRP3 inflammasome activation. Nature 469, 221-225

65. Nakahira, K. et al. (2011) Autophagy proteins regulate innate immune responses by inhibiting the release of mitochondrial DNA mediated by the NALP3 inflammasome. Nat. Immunol. 12, 222-230

66. Elliott, E.I. et al. (2018) Cutting edge: mitochondrial assembly of the NLRP3 inflammasome complex is initiated at priming. I. Immunol. 200, 3047-3052

67. Shimada, K. et al. (2012) Oxidized mitochondrial DNA activates the NLRP3 inflammasome during apoptosis. Immunity 36 401-414

68. Zhong, Z. et al. (2018) New mitochondrial DNA synthesis enables NLRP3 inflammasome activation. Nature 560, 198-203

69. Hornung, $V$ et al. (2009) AlM2 recognizes cytosolic dsDNA and forms a caspase-1-activating inflammasome with ASC. Nature 458, 514-518

70. Fernandes-Alnemri, T. et al. (2009) AlM2 activates the inflammasome and cell death in response to cytoplasmic DNA. Nature 458, 509-513

71. Dang, E.V. et al. (2017) Oxysterol restraint of cholesterol synthesis prevents AIM2 inflammasome activation. Cell 171 , 1057-1071.e11

72. West, A.P. et al. (2011) Mitochondria in innate immune responses. Nat. Rev. Immunol. 11, 389-402

73. Mostowy, S. and Shenoy, A.R. (2015) The cytoskeleton in cellautonomous immunity: structural determinants of host defence. Nat. Rev. Immunol. 15, 559-573

74. Underhill, D.M. and Goodridge, H.S. (2012) Information processing during phagocytosis. Nat. Rev. Immunol. 12, 492-502

75. Man, S.M. et al. (2014) Actin polymerization as a key innate immune effector mechanism to control Salmonella infection. Proc. Natl. Acad Sci. U. S. A. 111, 17588-17593

76. Flannagan, R.S. et al. (2012) The cell biology of phagocytosis. Annu. Rev. Pathol. 7, 61-98

77. Yates, R.M. et al. (2005) The kinetics of phagosome maturation as a function of phagosome/lysosome fusion and acquisition of hydrolytic activity. Traffic $6,413-420$

78. Chan, M.P. et al. (2015) DNase II-dependent DNA digestion is required for DNA sensing by TLR9. Nat. Commun. 6, 5853

79. Wu, J. et al. (2013) Cyclic GMP-AMP is an endogenous second messenger in innate immune signaling by cytosolic DNA. Science 339, 826-830

80. Turley, S.J. et al. (2000) Transport of peptide-MHC class II complexes in developing dendritic cells. Science 288, 522-527

81. Inaba, K. et al. (2000) The formation of immunogenic major histocompatibility complex class II-peptide ligands in lysosomal 
compartments of dendritic cells is regulated by inflammatory stimuli. J. Exp. Med. 191, 927-936

82. Brown, G.D. et al. (2003) Dectin-1 mediates the biological ef fects of beta-glucans. J. Exp. Med. 197, 1119-1124

83. Gantner, B.N. et al. (2003) Collaborative induction of inflammatory responses by dectin-1 and Toll-like receptor 2. J. Exp. Med. 197, 1107-1117

84. Kagan, J.C. et al. (2008) TRAM couples endocytosis of Toll-like receptor 4 to the induction of interferon-beta. Nat. Immunol. 9, 361-368

85. Sasai, M. et al. (2010) Bifurcation of Toll-like receptor 9 signaling by adaptor protein 3. Science 329, 1530-1534

86. Man, S.M. et al. (2014) Inflammasome activation causes dua recruitment of NLRC4 and NLRP3 to the same macromolecular complex. Proc. Natl. Acad. Sci. U. S. A. 111, 7403-7408

87. Keestra, A.M et al (2013) Manipulation of small Rho GTPases is a pathogen-induced process detected by NOD1. Nature 496, 233-237

88. Bielig, $\mathrm{H}$. et al. (2014) The cofilin phosphatase slingsho homolog 1 (SSH1) links NOD1 signaling to actin remodeling, In PLoS Pathog, p. e1004351

89. Legrand-Poels, S. et al. (2007) Modulation of Nod2-dependent NF-kappaB signaling by the actin cytoskeleton. J. Cell Sci. 120, 1299-1310

90. Fukazawa, A. et al. (2008) GEF-H1 mediated control of NOD1 dependent NF-kappaB activation by Shigella effectors. PLoS Pathog. 4, e1000228

91. Zhao, Y. et al. (2012) Control of NOD2 and Rip2-dependent innate mmune activation by GEF-H1. Inflamm. Bowel Dis. 18, 603-612

92. $\mathrm{Xu}, \mathrm{H}$. et al. (2014) Innate immune sensing of bacterial modifications of Rho GTPases by the pyrin inflammasome. Nature 513, 237-241

93. Park, Y.H. et al. (2016) Pyrin inflammasome activation and RhoA signaling in the autoinflammatory diseases FMF and HIDS. Nat. Immunol. 17, 914-921

94. Dinarello, C.A. et al. (1974) Colchicine therapy for familial Mediterranean fever. A double-blind trial. N. Engl. J. Med. 291, 934-937

95. Ahrens, S. et al. (2012) F-actin is an evolutionarily conserved damage-associated molecular pattern recognized by DNGR1, a receptor for dead cells. Immunity $36,635-645$

96. Zhang, J.G. et al. (2012) The dendritic cell receptor Clec9A binds damaged cells via exposed actin filaments. Immunity $36,646-657$

97. Shi, H. et al. (2016) NLRP3 activation and mitosis are mutually exclusive events coordinated by NEK7, a new inflammasome component. Nat. Immunol. 17, 250-258

98. Misawa, T. et al. (2013) Microtubule-driven spatial arrangement of mitochondria promotes activation of the NLRP3 inflammasome. Nat. Immunol. 14, 454-460

99. Zeng, Q.Z. et al. (2019) Paclitaxel enhances the innate immunity by promoting NLRP3 inflammasome activation in macrophages. Front. Immunol. 10, 72
100. Henry, E. et al. (2013) Recognition of bacterial plant pathogens: local, systemic and transgenerational immunity. New Phytol. 199, 908-915

101. Maekawa, T. et al. (2011) NLR functions in plant and animal immune systems: so far and yet so close. Nat. Immunol. 12 , $817-826$

102. Jones, J.D. et al. (2016) Intracellular innate immune surveillance devices in plants and animals. Science 354. Published online December 2, 2016. https://doi.org/10.1126/science.aaf6395

103. Netea, M.G. et al. (2016) Trained immunity: a program of innate immune memory in health and disease. Science 352, aaf1098

104. Biswas, S.K. and Mantovani, A. (2010) Macrophage plasticity and interaction with lymphocyte subsets: cancer as a paradigm. Nat. Immunol. 11, 889-896

105. Bowdish, D.M. et al. (2007) Macrophage receptors implicated in the "adaptive" form of innate immunity. Microbes Infect. 9 , $1680-1687$

106. Foster, S.L. et al. (2007) Gene-specific control of inflammation by TLR-induced chromatin modifications. Nature 447, 972-978

107. Spoel, S.H. and Dong, X. (2012) How do plants achieve immunity? Defence without specialized immune cells. Nat. Rev. Immunol. 12, 89-100

108. Shine, M.B. et al. (2019) Signaling mechanisms underlying systemic acquired resistance to microbial pathogens. Plant Sci. $279,81-86$

109. Fine, N. et al. (2016) Role of the cytoskeleton in myeloid cell function. Microbiol. Spectr. https://doi.org/10.1128/ microbiolspec.MCHD-0029-2016

110. Janke, C. and Montagnac, G. (2017) Causes and consequences of microtubule acetylation. Curr. Biol. 27, R1287-R1292

111. Stradal, T.E.B. and Schelhaas, M. (2018) Actin dynamics in host-pathogen interaction. FEBS Lett. 592, 3658-3669

112. Aktories, K. et al. (2011) Actin as target for modification by bacterial protein toxins. FEBS J. 278, 4526-4543

113. Lang, A.E. et al. (2017) Actin ADP-ribosylation at threonine148 by Photorhabdus luminescens toxin TccC3 induces aggregation of intracellular F-actin. Cell Microbiol. Published online July 15, 2016. http://dx.doi.org/10.1111/cmi.12636

114. Kudryashova, E. et al. (2018) Actin cross-linking toxin is a universal inhibitor of tandem-organized and oligomeric g-actin binding proteins. Curr. Biol. 28, 1536-1547.e9

115. Bozhokina, E.S. et al. (2011) Bacterial invasion of eukaryotic cells can be mediated by actin-hydrolysing metalloproteases grimelysin and protealysin. Cell Biol. Int. 35, 111-118

116. Liu, Y. et al. (2017) A Legionella effector disrupts host cytoskeletal structure by cleaving actin. PLoS Pathog. 13, e1006186

117. Wilkinson, A.W. et al. (2019) SETD3 is an actin histidine methyltransferase that prevents primary dystocia. Nature $565,372-376$

118. Dangl, J.L. and Jones, J.D.G. (2001) Plant pathogens and integrated defence responses to infection. Nature 411, 826-833

119. Andree, M. et al. (2014) BID-dependent release of mitochondrial SMAC dampens XIAP-mediated immunity against Shigella. EMBO J. 33, 2171-2187 